\title{
The (in)validity of benefit transfer and its consequences for policy-making
}

\author{
E. J. Bos ${ }^{1} \&$ J. M. Vleugel ${ }^{2}$ \\ ${ }^{1}$ LEI, The Netherlands \\ ${ }^{2}$ OTB Research Institute, TU Delft, The Netherlands
}

\begin{abstract}
The essence of benefit transfer is that the assessment of ecological effects of an intended project is based on information from similar studies, undertaken at other sites. Instead of performing a completely new study, which may cost a lot of time and money, key parameters from older studies are used. This approach is assumed to save valuable research time and money. In the paper it will be shown that this benefit may occur at the expense of the validity of assessments. Using these assessments for the new project may result in sub-optimal decision-making by governments and other stakeholders, like private landowners. The paper contains a meta-analysis on the validity of benefit transfer. The two basic types of benefit transfer, simple benefit transfer and benefit function transfer are being compared. It turns out that the validity of simple benefit transfer is often poor even when applied at comparable sites. This raises the question whether simple benefit transfer should be used to support policy-making regarding investment scenarios of national importance. It may be better to use benefit function transfer as an alternative, because it can compensate for differences in explanatory variables. In this way, the validity of the assessment can be increased, which may lead to 'better' decisions, provided that suitable benefit functions are available.
\end{abstract}

Keywords: environmental economics, benefit transfer, validity, decision-making.

\section{Introduction}

The impact of an intended investment project, such as the development of a nature area, can be evaluated with two alternative methods of collecting data. The first option is called an in depth or full study and the second option is to take 
research results from other studies and apply them in a new study. This approach is referred to as benefit transfer. There are two main ways of carrying out a benefit transfer study. The first is called simple benefit transfer and the second function benefit transfer. Our aim in this paper is to compare the validity of these two benefit transfer methods using a simple meta-analytical framework.

The paper starts with an introduction of the importance of project assessment for decision-making. This is followed by a discussion of the aims of and the methodology employed in benefit transfer studies. Next, the two methods of benefit transfer will be compared. Based on these insights, suggestions will be formulated to contribute to a valid application of benefit transfer.

\section{Investment projects and policy-making}

Decision-making with respect to investment projects tends to be complex and extensive, because many, sometimes very diverging aims and interests have to be aligned. One of these is to protect the environment. This is especially true for decision-making on large infrastructure projects that have significant adverse effects on the environment. An assessment procedure is carried out. The result of such a procedure can be legally binding. In the Netherlands, for example, a MER (Environmental Impact Report) is obligatory for certain investment projects. It contains a non-monetary, partial analysis of the environmental consequences of a project. An integral assessment should cover economic, environmental, liveability, safety issues, et cetera. Dedicated project studies take place, which can be time and money consuming, especially if there is a major disagreement between proponents of the project and those opposing it. Opponents tend to use procedures in order to extend decision-making, hoping that this helps to delay and eventually to cancel the project.

An example is the Dutch 'Betuwelijn' case in the 1990-ties. This concerns the construction of a dedicated high-speed freight railway line of about 100 kilometres between Rotterdam (the largest harbour in Europe and one of the largest in the world) and the German border. Instead of using the existing railway line, which crosses through many cities, most of the freight trains will be relocated to this new railway line. This would enable a reduction of environmental and safety issues in urban areas and transport of much more freight by rail. The harbour of Rotterdam and these cities would gain, but rural areas in the Betuwe-region would be intersected. This is a problem because the Betuwe-region contains important ecological and culture-historical values. A compromise had to be found, which increased the decision-making period [1], while the building costs doubled due to compensating investments (noise shielding, bypass tunnels, et cetera).

In the past decade, the number of infrastructure projects has increased. At the same time, policy-makers want to reduce evaluation periods, which puts stress on researchers to speed up their work. This is why less time and money consuming ways of evaluating policy options have become interesting. The question is whether such assessment techniques enable a reliable support of decision-making on public investment projects. 


\section{Environmental assessment and benefit transfer}

\subsection{Introduction}

The aim of environmental assessment is to first assess an effect in physical terms and second, to translate this impact into monetary terms. The determination of physical effects is the field of ecologists. (Environmental) economists are involved in the economic valuation of the effects, i.e. the determination of costs and benefits of a project.

With respect to the economic valuation, a distinction can be made between performing an in depth study and applying benefit transfer. Performing an in depth study using techniques such as contingent valuation involves the design, testing and implementation of a survey. This way of economic valuation of environmental features is characterised by extensive costs of collecting data [2].

\subsection{The methodology of benefit transfer}

Benefit transfer is based on the assumption that preferences for similar environmental concerns in corresponding contexts are comparable [3]. Estimations from one site (the study site) are used for the assessment of another site (policy site). In case of assessing environmental impacts, this approach is referred to as environmental benefit transfer [4]. More specifically, benefit transfer can be defined as the use of monetary environmental values estimated at a study site for estimations of the same parameters at a policy site through market-based or non-market-based economic valuation techniques [5]. It is being applied in various natural resource policy contexts, ranging from water quality management, associated health risks and waste management, to forest management and even global ecosystems [6].

Benefit transfer is supposed to increase the efficiency of the policy evaluation process [7]. This explains why many environmental agencies in the world are attracted to this method of collecting data [8]. For instance, the U.S. Environmental Protection Agency (EPA) suggests the application of benefit transfer in its guidelines for CBA [9]. Subsequently, in the U.S. some prominent scenarios have been assessed by applying benefit transfer. An example is the application of benefit transfer in a cost benefit analysis of the Clean Air Act performed by the U.S. Environmental Protection Agency. In the Netherlands, the application of benefit transfer is suggested in institutionalised guidelines for CBA when exploring possible project scenarios [10].

Two basic types of benefit transfer are being distinguished. The first is the direct or simple transfer of mean benefit estimates from study site to policy site [11]. To perform a simple benefit transfer, a researcher must make a number of subjective professional judgements such as the selection of the study site [3]. In order to reduce potential sources of measurement error associated with these judgements, a researcher should follow three criteria when considering the use of an existing study [12]: 
- The commodities of the policy site which are subject of evaluation should be comparable to those of the study site;

- The population characteristics of policy site and study site should be similar. This can be a hazardous case when commodities reflect non-use values: what is the relevant population for non-use of an area in the first place?

- A researcher has to use the welfare measurement from the study site, because he or she cannot transfer welfare measurements used in a willingness to pay context to a willingness to accept context and vice versa.

The second type is the transfer of functions rather than final benefits estimates (benefit function transfer [11]). It starts with the construction of a value function based on existing literature. Next, data have to be collected about independent variables at the policy site in order to estimate the benefit for the policy site.

\section{A comparison of benefit transfer methods: pros and cons}

Benefit transfer can be considered as a time and money reducing alternative for collecting economic data. Collecting economic data is especially costly when it concerns environmental goods and services such as biodiversity and amenities.

When applying simple benefit transfer a researcher has to find mean values from studies that have been performed for similar cases. The search for similar values should be based on the three criteria mentioned in section 3.2. It is up to the researcher to judge whether the values of the policy site correspond sufficiently to those of the study site. In other words, whether it ultimately makes sense to compare the two sites.

The key issue with benefit function transfer is that a researcher has to use an already determined relation between the variable of the commodity of interest and the corresponding explanatory variables. The problem is that in most cases a suitable function has not been determined. This is no surprise as most in depth studies primarily intend to assess mean benefits for the concerned case instead of assessing the functional relation between the environmental benefit and explanatory variables. Assessing a benefit function would only be efficient if this function could be used for several additional studies in future...

In case a suitable function could be found, the next step is to collect data for the explanatory variables. This may again be a difficult step. Consider for instance benefit assessment of non-use values. An example of a non-use value is the benefit of the protection of biodiversity in a nature area that is closed for users like recreational visitors. To determine such a value it is necessary to collect social-economic data (age, income, education, et cetera). This means that the researcher has to know the population for whom these non-use values are relevant. It is very arbitrary to determine which people belong to the relevant population and who should be excluded. Hence, collecting data for a benefit function transfer might be less obvious than it seems. 
This means that it may take more time and money to carry out a benefit function transfer study instead of a simple benefit transfer study. What pleads in favour of benefit function transfer according to several authors, is that more information is effectively transferred $[5,8]$. In particular, benefit function transfer enables a more explicit correction for explanatory variables, which means that its validity is likely to be higher, provided that proper functions are available. In other words, within the context of benefit transfer there is a tradeoff between validity and costs of collecting data. Therefore, in the following section we will address the validity of both benefit transfer methods.

\section{Does benefit transfer produce valid outcomes?}

\subsection{An overview of validity studies}

In this section we will discuss the validity of benefit (function) transfer with a number of examples from the literature. As will be shown, there is a major difference of opinion about the validity of benefit transfer. There are even authors who say that the method is of no value at all.

A pioneering study was the one by Loomis in 1992 [13]. The author tested the transferability of travel cost demand equations and contingent valuation benefit functions for recreational fishing in the United States. Transferring these functions from one state to another gave accurate results. However, transferring a function from a recreation site in one state to another state gave invalid estimates. It appears that specific regional aspects determine the concerned benefit in this study.

Another interesting study was carried out by Bergland et al. in 1995 [8]. It estimated benefits functions for similar environmental goods by means of parallel contingent valuation (CVM) studies conducted at two Norwegian water sites. The authors tested the validity of simple benefit transfer and benefit functions transfer empirically. Neither type of benefit transfer gave valid values. Moreover, they concluded that the two benefit functions derived from the CVM studies were not related.

In 1996 a first review of benefit transfer studies was carried out by Bergstrom in the U.S. [14]. Although the studies involved in this review suggest that benefit function transfer may be more valid than simple benefit transfer, the validity of neither form of benefit transfer is strongly supported by empirical tests. Bergstrom concludes that it is a challenge to determine adjustment procedures and protocols, which lead to accurate estimates.

Bhat et al. [3] have examined the transferability of consumer surplus estimates (simple benefit transfer) and the transferability of benefit functions from the Southern Appalachian Mountain 'ecoregion' to a local site within the ecoregional level. They found that transferability of benefit estimates from recreational activities was possible in $50 \%$ of cases, whereas benefit functions could be transferred for all the activities considered in their study. The results of their study suggest that benefit transfer between sites located in a similar natural 
area or region generates valid estimates. In order to improve the validity of benefit transfer, these authors recommend further research both in estimation procedures and accounting for explanatory variables like site attributes.

The need to include site characteristics is also emphasized by Bowker et al. [15]. Moreover, these authors show that transferring benefit functions, which are derived from multi-site data is most promising as it allows for correction of differences in site characteristics.

These positive findings are in contrast with those by Brouwer [5] who concludes that empirical testing of environmental benefit transfer has not lead to valid results so far.

Kristofersson et al. [16] show that the validity of benefit transfer is likely to be even less than found in earlier validity studies. They suggest that validity tests are biased towards positive outcomes and therefore provide no accurate information.

Finally, Brander and Florax [17] conclude on the basis of studies performed in the U.S. and the U.K. that the validity of benefit transfer is disappointing. More specifically, they found that simple benefit transfer can generate transfer errors of up to $400 \%$.

Given these insights it not surprisingly that Florax et al. [18] notice that despite the intuitive appeal of benefit transfer, its application in environmental assessment studies is very limited. The risk of using poor data is that the outcome of CBA might result in sub-optimal decision-making. In that case the cost-reducing feature of benefit transfer might be out-weighted by the social cost of sub-optimal decision-making. This may prohibit the use of benefit transfer in any phase of policy making. The problem is that there are circumstances where benefit transfer has to be applied. This especially holds for ex ante evaluations, because in this case the effects have not yet taken place. Consider for instance the assessment of recreational benefits of a to be developed site using the travel costs method.

As there are no travellers to the site yet, travelling behaviour cannot be observed and travel cost cannot be determined. This prohibits the application of the travel costs method as an in depth study instrument. As in such circumstances benefit transfer has to be applied, it is important that reliable benefits assessments become available.

Table 1 summarizes the earlier studies.

\subsection{A spatial frontier?}

The studies discussed above indicate that only intra-regional benefit function transfer will yield valid estimates. Consequently, interregional, interstate-, international- and intercontinental transfers are likely to generate invalid estimates.

This also means that if in a certain country no previous valuation studies have been carried out, then it is hardly possible to apply a valid benefit transfer. This case applies to the Netherlands, for instance. The current body of benefit estimates in this country largely consists of data from abroad [19]. The relatively few studies that have been assessed in the Netherlands concern mean benefit 
estimates [20]. Benefit functions have not been assessed in any Dutch valuation study. As the present body of data lacks benefit functions for Dutch valuation studies, a valid application of benefit transfer in the Netherlands is hardly feasible at the moment.

It follows that a more valid application of benefit transfer in the Netherlands is likely to occur when benefit functions are being assessed in order to enable benefit function transfer. Up till then, one should only perform in depth studies and not apply benefit transfer for supporting Dutch decision-making.

Table 1: $\quad$ Meta-analysis of existing benefit transfer studies.

\begin{tabular}{|l|l|l|}
\hline Study & Simple & Function \\
\hline Loomis [13] & Problematic & Valid \\
\hline Bergland et al. [8] & Problematic & Problematic \\
\hline Bergstrom [14] & Problematic & Problematic \\
\hline Bhat et al. [3] & N.A. & Intraregional is valid \\
\hline Bowker et al. [15] & N.A. & Possible \\
\hline Brouwer [5] & Problematic & Problematic \\
\hline $\begin{array}{l}\text { Kristofersson et al. } \\
{[16]}\end{array}$ & Very problematic & Very problematic \\
\hline $\begin{array}{l}\text { Brander and Florax } \\
{[17]}\end{array}$ & Very problematic & Very problematic \\
\hline
\end{tabular}

\subsection{Scope for improvement}

If we consider the observation that an in depth study may be very costly in most cases, while a benefit transfer study may lead to doubts about the validity of its results in specific cases, there is an argument in favour of combining in depth studies and benefit transfer in the same study. One of the authors [21] who followed this route applied Bayesian statistics to a set of data from 31 case studies throughout the world. This statistical tool allowed him to generate data for additional explanatory variables. A similar approach was followed by another author [22], who concluded that conventional benefit transfer based on CVM underestimated willingness to pay by about $50 \%$. She concluded that Bayesian statistics saved on study costs, while its results are much more valid.

It follows, that deriving benefit functions using Bayesian statistics can combine the best of benefit transfer and in depth, i.e. efficiency and validity.

\section{Conclusions and recommendations}

This paper discussed the application of benefit transfer in project assessment studies. Benefit transfer is an interesting alternative for in depth studies, because it might reduce research time and cost considerably.

However, the burden of benefit transfer might come in the form of invalid assessments. If the difference between the estimated and real values becomes too 
large, then the validity of decision making based on such estimations is questionable.

In this article a comparison of the validity of two main benefit transfer methods, simple and function benefit transfer has been presented. Some authors, who criticize simple benefit transfer, recommend benefit function transfer as the most valid of two. The latter is regarded as a more accurate alternative for simple benefit transfer, because more information is effectively transferred. However, suitable benefit functions are hardly available. Studies show that socio-cultural parameters prohibit the use of regional values and estimates of such values beyond the regional level. This means that interregional, interstate or international transfers are not likely to lead to valid results.

A promising alternative is a combination of a limited in depth study and a database with values from a range of international studies using Bayesian statistics. The local in depth study can then be used to validate the international assessment values.

As became apparent, more research should be carried out in the following directions:

- Assessment of more transferable benefit functions;

- Perform comparative studies to analyse the validity and costs of both benefit transfers and in depth studies;

- Combining in-depth studies with benefit transfer in order to profit from the strong points of both methods.

\section{References}

[1] Boom, H. \& Metze, M., Slag om de Betuweroute; Het Spel langs de Lijn, 1997.

[2] Barbier, E. B., Acreman, M. \& Knowler, D., Economic Valuation of Wetlands, Ramsar Convention Bureau. Imprimerie Dupuis, S.A., Le Brassus, Switzerland, 1997.

[3] Bhat, G., Bergstrom, J.C. \& Bowker, M.J., An Ecoregional Approach to Benefit Transfer, Discussion paper, 1997.

[4] Kask, S. B. \& Shogren, J.F., Benefit Transfer Protocol for Long-term Health Risk Valuation; A Case of Surface Water Contamination, Water Resources Research, (30), pp. 2813-2824, 1994.

[5] Brouwer, R., Environmental value transfer; state of the art and future prospects, Ecological Economics, (32), pp. 137-152, 2000.

[6] Brouwer, R., Environmental value transfer: state of the art and future prospects, in: Florax, R., Nijkamp, P. \& and K. Willis, K., Comparative Environmental Economic Assessment. Edward Algar, Cheltenham, pp. 90-114, 2002.

[7] Button, K.J. \& Nijkamp, P., Environmental Policy Assessment and the Usefulness of Meta-Analysis. Socio-Economic Planning Sciences, (30), pp. 231-240, 1997.

[8] Bergland, O., Magnussen, K., \& Navrud, S., Benefit transfer: testing for accuracy and reliability, Discussion Paper \#D-03/1995, 1995. 
[9] See http://www.epa.gov/oar/sect812/1990-2010/chap1130.pdf.

[10] Ministerie van Landbouw, Natuur en Voedselveiligheid, Kentallen Waardering Natuur, Water, Bodem en Landschap: Hulpmiddel bij MKBA's. Eerste editie, Den Haag, 2006.

[11] Kirchhoff, S., Colby, B.G. \& LaFrance, J.T., Evaluating the performance of benefit transfer; an empirical inquiry, Journal of environmental economics and management, (33), pp. 75-93, 1997.

[12] Boyle, K. and \& Bergstrom, J.C., Benefit Transfers Studies: Myths, Pragmatism and Idealism, Water Resources Research, (28), pp. 657-663, 1992.

[13] Loomis, J. B., The evolution of a more rigorous approach to benefit transfer: benefit function transfer, Water Resource Research, (28), pp. 701-705, 1992.

[14] Bergstrom, J.C., Current Status of Benefits Transfer in the U.S.: a Review, 1996.

[15] Bowker, J.M., English, D.B.K. \& Bergstrom, J.C., Benefits Transfer and Count Data Travel Cost Models: an Application and Test of a Varying Parameter Approach with Guided Whitewater Rafting. FS 97-03, 1997.

[16] Kristofersson, D. \& Navrud, S., Validity Tests of Benefit Transfer - Are we Perfoming the Wrong Tests? Environmental and Resource Economics, (30), pp. 279-286, 2005.

[17] Brander, L.M. and Florax, J.G.M., The valuation of wetlands: primary versus meta-analysis based value transfer. In Carruthers, J.I. \& Mundy, B. (eds.), Environmental Valuation: Interregional and Intraregional Perspectives. Aldershot: Ashgate (in press).

[18] Florax, R., Nijkamp, P. \& and K. Willis, K., Comparative Environmental Economic Assessment. Edward Algar, Cheltenham, 2002.

[19] See the "Environmental Valuation Reference Inventory" (EVRI) at http://www.evri.ec.gc.ca/evri/english/about.htm.

[20] Ruijgrok, E.C.M., Valuation of Nature and Environment: A historical overview of Dutch socio-economic valuation studies, Platform voor Economische Waardering van Natuur, Rotterdam, 2002.

[21] Bal, F., Valuing Dutch Nature Areas: a Different Approach, Ph. D. Thesis. Martin Luther University, Halle/Wittenberg, 2002.

[22] Lehr, U., Bayesian benefit transfer in environmental evaluation, University of Hohenheim, Dpt. of Economics, Stuttgart, 2005. 